Communications in Physics, Vol.20, No. 2 (2010), pp. 113-119

\title{
FIELD EQUATIONS IN GENERAL RELATIVITY WITH INTERNAL SYMMETRY
}

\author{
DO NGOC DIEP \\ Institute of Mathematics, VAST \\ DAO VONG DUC, HA VINH TAN, AND NGUYEN AI VIET \\ Institute of Physics, VAST
}

\begin{abstract}
The covariant wedge products of tetrads in General Relativity are introduced and related field equations are derived. The electromagnetic dual field is treated in detail and it is shown that this field is closely related with one component field, which is eigenfunction of d'Alembertian operator. The formalism is developed to incorporate internal symmetry.
\end{abstract}

\section{INTRODUCTION}

The construction of the unified theory of all fundamental interactions has been an enthusiastic source for intensive research works during the last decades. Notable progress has been made in some directions, among those are string theories $[1,2]$ and standard models $[3,4]$. However, they all have their own complexities and shortcomings, and most important, neither can combine Einstein's General Relativity and Quantum Theory in a suitable manner.

Recently, on the other hand, there has been a lot of works, e.g. [5-8], making attempts to approach the unification problem on the fundamental of General Relativity. This idea is also in a perfect agreement with the Geometric Langlands Correspondence $[9,10]$ between Geometry and Physics. The essence of the insights is that all fundamental interactions are originated from the nature of spacetime itself, as the gravitational is.

Our present work is done along this line. Its aim is to elaborate on the concept of wedge products for tetrad and to derive the related equations. The paper is organized as follows. In Sec. 2 the wedge products with respect to tetrad and Lorentz indices are introduced, as well as the generally covariant version of $\epsilon^{\mu \nu \lambda \rho}$, which we name $B$-field. The related field equations are derived in Sec. 3. In particular, it is shown that the $B$ field is eigenfunction of d'Alembertian operator. Sec. 4 is devoted to the electromagnetic dual field. In the last section (Sec. 5) we extend the formalism to incorporate internal symmetry.

\section{COVARIANT WEDGE PRODUCTS FOR TETRAD}

The tetrad specifies a tangent space at each point of the Riemann manifold. E. Cartan found that the moving frame tetrad is an alternative way to describe Einstein's General Relativity. The tetrad denoted by $q_{\mu}^{a}(x)$ consists of four linear vectors satisfying 
the orthgonal relations:

$$
g^{\mu \nu}(x) q_{\mu}^{a}(x) q_{\nu}^{b}(x)=\eta^{a b},
$$

$a, b$ being tetrad indices, $\mu, \nu$ - Lorentz indices, $\eta^{a, b}$ - Minkowski metric $=\operatorname{diag}(1,-1,-1,-1)$, $g^{\mu \nu}(x)$ - spacetime metric tensor.

The meaning of tetrad is given from the reciprocal formula

$$
\eta_{a b} q_{\mu}^{a}(x) q_{\nu}^{b}(x)=g_{\mu \nu}(x)
$$

which means that the metric tensor can be expressed in terms of tetrad.

The tetrad components of an arbitrary tensor $T \mu_{1} \mu_{2} \ldots \mu_{r}{ }^{\nu_{1} \nu_{2} \ldots \nu_{s}}$ are defined as

$$
T_{a_{1} a_{2} \ldots a_{r}}^{b_{1} b_{2} \ldots b_{s}}(x) \equiv q_{a_{1}}^{\mu_{1}} \ldots q_{a_{r}}^{\mu_{r}} T_{\mu_{1} \mu_{2} \ldots \mu_{r}}^{\nu_{1} \nu_{2} \ldots \nu_{s}} q_{\nu_{1}}^{b_{1}} \ldots q_{\nu_{s}}^{b_{s}},
$$

which is invariant under the general transformation of spacetime coordinates

$$
x^{\mu} \rightarrow x^{\prime \mu}=f^{\mu}(x)
$$

In the tetrad formalism the invariance principle requires the action to be invariant under the general transformation (4) and the local Lorentz transformation of the tetrad:

$$
q_{a}^{\mu}(x) \rightarrow q_{a}^{\prime \mu}=\Lambda_{a}^{b}(x) q_{b}^{\mu}(x),
$$

where $\Lambda_{a}^{b}(x)$ are the local parameters satisfying the condition

$$
\eta^{a b} \Lambda_{a}^{c}(x) \Lambda_{b}^{d}(x)=\eta^{c d},
$$

The wedge products of tetrads are defined in the following manner:

- Wedge product $\wedge_{T}$ with respect to tetrad indices:

$$
G_{\mu \nu, a b}(x) \equiv\left(q_{\mu} \wedge_{T} q_{\nu}\right)_{a b}=\epsilon_{a b c d} q_{\mu}^{c}(x) q_{\nu}^{d}(x),
$$

where $\epsilon_{a b c d}$ is totally antisymmetric constant tensor with $\epsilon_{0123}=+1$;

- Wedge product $\wedge_{L}$ with respect to Lorentz indices:

$$
F^{\mu \nu, a b}(x)=\left(q^{a} \wedge_{L} q^{b}\right)^{\mu \nu}=\varepsilon^{\mu \nu \lambda \rho}(x) q_{\lambda}^{a}(x) q_{\rho}^{b}(x),
$$

where $\varepsilon^{\mu \nu \lambda \rho}(x)$ is totally antisymmetric tensor having $\epsilon^{a b c d}$ as tetrad components, namely

$$
\varepsilon^{\mu \nu \lambda \rho}(x) \equiv \epsilon^{a b c d} q_{a}^{\mu}(x) q_{b}^{\nu}(x) q_{c}^{\lambda}(x) q_{d}^{\rho}(x)
$$

and

$$
\epsilon^{a b c d} \equiv q_{\mu}^{a}(x) q_{\nu}^{b}(x) q_{\lambda}^{c}(x) q_{\rho}^{d}(x) \varepsilon^{\mu \nu \lambda \rho}(x) .
$$

Note that $G_{\mu \nu, a b}(x)$ and $F^{\mu \nu \lambda \rho}(x)$ are antisymmetric with respect both to $\mu \nu$ and $a b, \varepsilon^{\mu \nu \lambda \rho}(x)$ is totally antisymmetric.

- Wedge product with respect both to tetrad and Lorentz indices:

$$
T_{a b}^{\mu \nu}(x) \equiv\left(q \wedge_{L T} q\right)_{a b}^{\mu \nu}=\varepsilon^{\mu \nu \lambda \rho}(x) \epsilon_{a b c d} q_{\lambda}^{c}(x) q_{\rho}^{d}(x) .
$$




\section{BASIC EQUATIONS}

In this section we are dealing with some basic equations related to tetrad.

First it is worth noting that the Einstein's equation

$$
R_{\mu \nu}-\frac{1}{2} R g_{\mu \nu}=k T_{\mu \nu}
$$

and the equation for tetrad

$$
\left(R_{\mu}^{\nu}-\frac{1}{2} R \delta_{\mu}^{\nu}-k T_{\mu}^{\nu}\right) q_{\nu}^{a}(x)=0
$$

are quite equivalent. This can be easily shown, using equations (1) and (2).

Next we derive the equations for the wedge products defined in $\S 2$. The starting point is the equation for covariant derivative of tetrad:

$$
D_{\alpha} q_{\mu}^{a} \equiv \partial_{\alpha} q_{\mu}^{a}(x)-\Gamma_{\alpha \mu}^{\nu}(x) q_{\nu}^{a}(x)=0,
$$

$\Gamma_{\alpha \mu}^{\nu}(x)$ being affine connection. This equation is a direct consequence of the equation

$$
D_{\alpha} g_{\mu \nu}(x)=0
$$

and equation (2).

Let $V^{\mu \nu}$ be some antisymmetric tensor having the form of tetrad product like (7)(11). It follows immediately that

$$
D_{\alpha} V^{\mu \nu}(x) \equiv \partial_{\alpha} V^{\mu \nu}+\Gamma_{\alpha \sigma}^{\mu} V^{\sigma \nu}+\Gamma_{\alpha \sigma}^{\nu} V^{\mu \sigma}=0
$$

and as a result we have

$$
\partial_{\mu} V^{\mu \nu}=-\Gamma_{\mu \sigma}^{\sigma} V^{\mu \nu}
$$

Hence, we have the equations:

$$
\begin{gathered}
\left(\partial_{\mu}+\Gamma_{\mu \sigma}^{\sigma}\right) G_{a b}^{\mu \nu}(x)=0 \\
\left(\partial_{\mu}+\Gamma_{\mu \sigma}^{\sigma}\right) F^{\mu \nu, a b}(x)=0 \\
\left(\partial_{\mu}+\Gamma_{\mu \sigma}^{\sigma}\right) T_{a b}^{\mu \nu}(x)=0
\end{gathered}
$$

Taking the differentiation $\partial_{\nu}$ of both sides of equation (17), we have

$$
\begin{aligned}
& 0=-\partial_{\nu} \Gamma_{\mu \sigma}^{\sigma} V^{\mu \nu}-\Gamma_{\mu \sigma}^{\sigma} \partial_{\nu} V^{\mu \nu}= \\
& =-\partial_{\nu} \Gamma_{\mu \sigma}^{\sigma} V^{\mu \nu}+\Gamma_{\mu \sigma}^{\sigma} \Gamma_{\nu \rho}^{\rho} V^{\mu \nu}=-\partial_{\nu} \Gamma_{\mu \sigma}^{\sigma} V^{\mu \nu} \text {. }
\end{aligned}
$$

Hence,

$$
\begin{aligned}
\partial_{\nu} \Gamma_{\mu \sigma}^{\sigma} F^{\mu \nu, a b} & =0 \\
\partial_{\nu} \Gamma_{\mu \sigma}^{\sigma} G_{a b}^{\mu \nu} & =0 \\
\partial_{\nu} \Gamma_{\mu \sigma}^{\sigma} T_{a b}^{\mu \nu} & =0
\end{aligned}
$$

For $\varepsilon_{\mu \nu \lambda \rho}(x)$ tensor the equation

$$
D_{\alpha} \varepsilon_{\mu \nu \lambda \rho}(x)=0
$$

gives

$$
\begin{aligned}
\partial_{\alpha} \varepsilon_{\mu \nu \lambda \rho}(x) & =\Gamma_{\alpha \mu}^{\sigma} \varepsilon_{\sigma \nu \lambda \rho}(x)+\Gamma_{\alpha \nu}^{\sigma} \varepsilon_{\mu \sigma \lambda \rho}(x)+\Gamma_{\alpha \lambda}^{\sigma} \varepsilon_{\mu \nu \sigma \rho}(x)+\Gamma_{\alpha \rho}^{\sigma} \varepsilon_{\mu \nu \lambda \sigma}(x) \\
& =\Gamma_{\alpha \sigma}^{\sigma} \varepsilon_{\mu \nu \lambda \rho}(x)
\end{aligned}
$$


Similarly, we have

$$
\partial_{\alpha} \varepsilon^{\mu \nu \lambda \rho}(x)=-\Gamma_{\alpha \sigma}^{\sigma} \varepsilon^{\mu \nu \lambda \rho}(x) .
$$

Taking the differentiation $\partial^{\alpha} \equiv \eta^{\alpha \beta} \partial_{\beta}$ of both sides of equations (22) and (23), we have

$$
\left\{\square-\left(\eta^{\alpha \beta} \Gamma_{\alpha \gamma}^{\gamma} \Gamma_{\beta \delta}^{\delta}+\partial^{\alpha} \Gamma_{\alpha \gamma}^{\gamma}\right)\right\} \varepsilon_{\mu \nu \lambda \rho}(x)=0
$$

and

$$
\left\{\square-\left(\eta^{\alpha \beta} \Gamma_{\alpha \gamma}^{\gamma} \Gamma_{\beta \delta}^{\delta}-\partial^{\alpha} \Gamma_{\alpha \gamma}^{\gamma}\right)\right\} \varepsilon^{\mu \nu \lambda \rho}(x)=0
$$

where $\square=\eta^{\alpha \beta} \partial_{\alpha} \partial_{\beta}$ is the d'Alembertian operator, $\eta^{\alpha \beta}$ - the Minkowski metric tensor.

So, $\varepsilon^{\mu \nu \lambda \rho}(x)$ tensor, which we name B-field,

$$
B(x)=\varepsilon^{0123}(x),
$$

is eigenfunction of d'Alembertian operator.

\section{ELECTROMAGNETIC DUAL FIELD}

Consider now the electromagnetic field strength tensor

$$
F_{\mu \nu} \equiv \partial_{\mu} A_{\nu}-\partial_{\nu} A_{\mu}
$$

First we note that $F_{\mu \nu}$ is a generally covariant tensor because the affine connection $\Gamma_{\mu \nu}^{\sigma}$ is symmetric, $\Gamma_{\mu \nu}^{\sigma}=\Gamma_{\nu \mu}^{\sigma}$, and hence we can rewrite it in terms of covariant derivatives:

$$
F_{\mu \nu}=D_{\mu} A_{\nu}-D_{\nu} A_{\mu} .
$$
(8):

Define the wedge product $\wedge_{L}$ of $D_{\mu}$ and $A_{\mu}$ according to the formula analogous to

$$
\begin{aligned}
B^{\mu \nu}(x) & \equiv\left(D \wedge_{L} A\right)^{\mu \nu} \equiv \varepsilon^{\mu \nu \lambda \rho}(x) D_{\lambda} A_{\rho}(x) \\
& =\frac{1}{2} \varepsilon^{\mu \nu \lambda \rho}(x) F_{\lambda \rho}(x) .
\end{aligned}
$$

Let us remind that with the identification

$$
F_{0 i}=E_{i}, F_{23}=H_{1}, F_{31}=H_{2}, F_{12}=H_{3}
$$

for electric $\vec{E}$ and magnetic $\vec{H}$ fields, equation (27) represents Maxwell's equations:

$$
\begin{aligned}
\vec{E} & =-\operatorname{grad} A_{0}+\frac{\partial \vec{A}}{\partial t} \\
\vec{H} & =\operatorname{rot} \vec{A}
\end{aligned}
$$

The equation (29) gives the relations

$$
\begin{aligned}
& B^{0 i}(x)=B(x) H_{i}(x) \\
& B^{i j}(x)=\epsilon^{i j k} B(x) E_{k}(x) ; \quad i, j, k=1,2,3
\end{aligned}
$$

where $B(x)$ is defined in $(26), \epsilon^{123}=+1$. Now we look for the equation for $B^{\mu \nu}(x)$. From the definition (29) we have

$$
\partial_{\mu} B^{\mu \nu}(x)=\frac{1}{2}\left(\partial_{\mu} \varepsilon^{\mu \nu \lambda \rho}(x) F_{\lambda \rho}(x)+\varepsilon^{\mu \nu \lambda \rho} \partial_{\mu} F_{\lambda \rho(x)}\right)
$$


The second term in the right hand side vanishes identically, while the first term can be again expressed in terms of $B^{\mu \nu}$ using the equation (23) to give

$$
\left(\partial_{\mu}+\Gamma_{\mu \sigma}^{\sigma}\right) B^{\mu \nu}(x)=0 .
$$

The limiting case when the spacetime is flat corresponds to the values

$$
\begin{gathered}
g_{\mu \nu}=\eta_{\mu \nu}, \Gamma_{\mu \nu}^{\sigma}=0 \\
B(x)=1, B^{0 i}(x)=H_{i}(x), B^{i j}(x)=\epsilon^{i j k} E_{k} .
\end{gathered}
$$

In this case the equation (4.8) reduces to

$$
\partial \mu B^{\mu \nu}(x)=0
$$

giving the relation

$$
\frac{\partial \vec{H}}{\partial t}=\operatorname{rot} \vec{E}
$$

in accordance with (31).

\section{TETRAD WITH INTERNAL SYMMETRY}

In this section we extend the results obtained above to incorporate the internal symmetry. Suppose the algebra of the underlying symmetry groups $G$ consists of $n$ generators $I_{i}, i=1,2, \ldots, n$, with the commutation relations:

$$
\left[I_{i}, I_{j}\right]=i f_{i j k} I_{k} ; \quad i, j, k=1,2, \ldots, n
$$

$f_{i j k}$ being structural constants of the Lie algebra $\mathfrak{g}=\operatorname{Lie} G$.

Now each tetrad component bears an internal group index besides others to become $q_{\mu i}^{a}(x)$, and instead of the formulae (1), (2) and (9) we have:

$$
\begin{gathered}
g_{\mu \nu, i j}(x)=\eta_{a b} q_{\mu i}^{a}(x) q_{\nu j}^{b}(x), \\
g_{\mu \nu}(x) \equiv g_{\mu \nu, i i}=\eta_{a b} q_{\mu i}^{a}(x) q_{\nu i}^{b}(x), \\
\eta^{a b}=q_{\mu i}^{a}(x) q_{\nu i}^{b}(x) g^{\mu \nu}, \\
\varepsilon_{\mu \nu \lambda \rho}(x)=\equiv \frac{1}{3} \epsilon_{a b c d} \sigma_{i j k l} q_{\mu i}^{a}(x) q_{\nu j}^{b}(x) q_{\lambda k}^{c}(x) q_{\rho l}^{d}(x) \\
\sigma_{i j k l} \equiv\left(\delta_{i j} \delta_{k l}+\delta_{i k} \delta_{j l}+\delta_{i l} \delta_{j k}\right) .
\end{gathered}
$$

The following wedge products of tetrad can be introduced:

- Wedge product with respect to tetrad indices:

$$
G_{\mu \nu, a b, i j} \equiv\left(q_{\mu i} \wedge_{T} q_{\nu j}\right)_{a b}=\epsilon_{a b c d} q_{\mu i}^{c} q_{\nu j}^{d} ;
$$

- Wedge product with respect to Lorentz indices:

$$
F_{i j}^{\mu \nu, a b} \equiv\left(q_{i}^{a} \wedge_{L} q_{j}^{b}\right)^{\mu \nu}=\varepsilon^{\mu \nu \lambda \rho} q_{\lambda i}^{a} q_{\rho j}^{b}
$$


- Wedge product with respect to internal indices:

$$
H_{\mu \nu, i}^{a b} \equiv\left(q_{\mu}^{a} \wedge_{I} q_{\nu}^{b}\right)_{i}=f_{i j k} q_{\mu j}^{a} q_{\nu k}^{b} .
$$

- Wedge product with respect to tetrad and Lorentz indices:

$$
T_{a b, i j}^{\mu \nu} \equiv\left(q_{i} \wedge_{L T} q_{j}\right)_{a b}^{\mu \nu}=\varepsilon^{\mu \nu \lambda \rho}(x) \epsilon_{a b c d} q_{\lambda i}^{c} q_{\rho j}^{d}
$$

- Wedge product with respect to tetrad and internal indices:

$$
N_{\mu \nu, a b, i} \equiv\left(q_{\mu} \Lambda_{T I} q_{\nu}\right)_{a b, i}=\epsilon_{a b c d} f_{i j k} q_{\mu j}^{c} q_{\nu k}^{d}
$$

- Wedge product with respect to Lorentz and internal indices:

$$
M_{i}^{\mu \nu, a b} \equiv\left(q^{a} \wedge_{L I} q^{b}\right)_{i}^{\mu \nu}=\varepsilon^{\mu \nu \lambda \rho}(x) f_{i j k} q_{\lambda j}^{a} q_{\rho k}^{b} .
$$

The wedge product with respect to all kinds of indices defined as

$$
(q \wedge q)_{a b, i}^{\mu \nu} \equiv \varepsilon^{\mu \nu \lambda \rho} \varepsilon_{a b c d} f_{i j k} q_{\lambda j}^{c} q_{\rho k}^{d}
$$

identically vanishes.

Note also that $N_{\mu \nu, a b, i}$ is symmetric with respect to Lorentz indices $(\mu \nu)$, while $F_{i j}^{\mu \nu, a b}, T_{a b, i j}^{\mu \nu}, M_{i}^{\mu \nu, a b}$ and

$$
\begin{aligned}
G_{\mu \nu, a b} & \equiv \sum_{i} G_{\mu \nu, a b, i i} \\
H_{\mu \nu, i} & \equiv \sum_{a, b} \eta_{a b} H_{\mu \nu, i}^{a b}
\end{aligned}
$$

are antisymmetric.

As products of tetrads, the covariant derivatives of all of them vanish, and as a consequence we have:

$$
\left(\partial_{\mu}+\Gamma_{\mu \sigma}^{\sigma}\right) N_{a b, i}^{\mu \nu}=-\Gamma_{\mu \sigma}^{\nu} N_{a b, i}^{\mu \sigma}
$$

and

$$
\left(\partial_{\mu}+\Gamma_{\mu \sigma}^{\sigma}\right) A^{\mu \nu}=0, \quad \partial_{\nu} \Gamma_{\mu \sigma}^{\sigma} A^{\mu \nu}=0 .
$$

In the case $G=\mathrm{SU}(3)$ from tetrad we can also construct symmetric second rank tensors of the form

$$
\begin{aligned}
S_{\mu \nu, k} & \equiv d_{i j k} \eta_{a b} q_{\mu i}^{a} q_{\nu j}^{b} \\
S_{\mu \nu, i j} & \equiv d_{i j k} S_{\mu \nu, k}=d_{i j k} d_{m n k} \eta_{a b} q_{\mu n}^{a} q_{\nu m}^{b}
\end{aligned}
$$

where $d_{i j k}$ is totally symmetric constant $\mathrm{SU}(3)$ tensor appearing in the anticommutators of Gell-Mann matricies:

$$
\left\{\lambda_{i}, \lambda_{j}\right\}=2 d_{i j k} \lambda_{k}+\frac{4}{3} \delta_{i j}
$$

and satisfying the identity

$$
\sum_{l}\left(d_{i j l} f_{l k m}+d_{j k l} f_{l i m}+d_{k i l} f_{l j m}\right)=0 .
$$


Being symmetric, the tensors (56) satisfy the same equation as (54):

$$
\left(\partial_{\mu}+\Gamma_{\mu \sigma}^{\sigma}\right) S_{k}^{\mu \nu}=-\Gamma_{\mu \sigma}^{\nu} S_{k}^{\mu \sigma}
$$

With the internal symmetry hidden in spacetime we might consider the spacetime coordinates $x^{\mu}$ of the form:

$$
x^{\mu}=\sum_{i=1}^{n} x_{i}^{\mu} h_{i},
$$

where $\left\{x_{i}^{\mu}\right\}$ and $\left\{h_{i}\right\}$ are attributed to regular representation of $G$, i.e. obey the commutation relations:

$$
\begin{aligned}
{\left[I_{k}, x_{j}^{\mu}\right] } & =i f_{k j l} x_{l}^{\mu} \\
{\left[I_{k}, h_{j}\right] } & =i f_{k j l} h_{l}
\end{aligned}
$$

Now using (40), we can construct for $x_{i}^{\mu}$ invariant interval

$$
d S^{2}=g_{\mu \nu, i j} d x_{i}^{\mu} d_{j}^{\nu}
$$

and using (47), (50), we can construct invariant areas:

$$
\begin{gathered}
d H=H_{\mu \nu, i}\left(d x^{\mu} \wedge_{I} d x^{\nu}\right)_{i}=H_{\mu \nu, i} f_{i j k} d x_{j}^{\mu} d x_{k}^{\nu}, \\
d M=M_{i}^{\mu \nu}\left(d x \wedge_{L I} d x\right)_{\mu \nu, i}=M_{i}^{\mu \nu} \varepsilon_{\mu \nu \lambda \rho}(x) f_{i j k} d x_{j}^{\lambda} d x_{k}^{\rho} .
\end{gathered}
$$

In the case $G=\mathrm{SU}(3)$, using (56) we can construct an invariant interval of the form:

$$
d S^{2}=S_{\mu \nu, i j} d x_{i}^{\mu} d x_{j}^{\nu} .
$$

\section{ACKNOWLEDGMENTS}

The authors thank colleagues from the Institute of Physics and the Institute of Mathematics, Vietnam Academy of Science and Technology, for stimulating discussions. This work is supported in part by Vietnam National Project for Research in Fundamental Sciences.

\section{REFERENCES}

[1] M. B. Green, J. H. Schwartz, E. Witten, Superstring Theory, Cambridge University Press 1987.

[2] L. Brink, D. Fridan, A.M. Polyakov, Physics and Mathematics of Strings, World Scientific, 1990.

[3] S. Weinberg, Phys. Rev. Lett. 19 (1967) 1264.

[4] A. Salam, Proceedings 8th Nobel Symposium, Stockholm 1968.

[5] M. S. Cohen, Clifford Residues and Charge Quantization, arXiv: gr-qc/0207107.

[6] D. N. Diep, D. V. Duc, H. V. Tan, N. A. Viet, Tetrads as Fundamental Concept in Generally Covariant Unified Theory, GAP4, 2006.

[7] D. N. Diep, D. V. Duc, H. V. Tan, N. A. Viet, Comm. in Phys.: Supplement 17 (2007) 36-47.

[8] D. N. Diep, D. V. Duc, H. V. Tan, N. A. Viet, Convolution Wedge Products of Fields ICTP Preprint, IC/2007/056, Trieste, Italy 2007.

[9] E. Frenkel, Lectures on Langlands Program and Conformal Field Theory, arXiv: hep-th/0512172.

[10] A. Kapustin, E. Witten, Electric-magnetic Duality and the Geometric Langlands Program, arXiv: hep-th/0604151.

Received 15 November 2008. 